be tender to palpation, with limitation of movement. There are sometimes also objective changes in the hand, with redness, swelling, and stiffness, which may end in atrophy of the small muscles. These "frozen shoulder" and "shoulderhand" syndromes may develop at any time within a year of the attack and are of importance, since it has been estimated ${ }^{5}$ that over $10 \%$ of patients have some degree of disability in shoulder or hand after myocardial infarction.

\section{Family and Community}

The teaching of social medicine has sometimes lacked a clearly defined basis and has included instruction on unrelated topics for which no other place could be found in the curriculum. This results in neither the teacher nor the student knowing what is expected of him. Now a report ${ }^{1}$ recently issued by the Royal College of Physicians defines as the prime function of a department of social and preventive medicine the teaching of epidemiology and the medical needs of society. It makes a strong plea for every medical school to have such a department with a full-time head. The main ground for dissatisfaction with part-time direction is not that it is inconsistent with substantial teaching but that it does not lead to the research activities which are so important to the development of a good academic department.

With the increasing emphasis on community care it is essential for the undergraduate to be given training in the approach to medical problems by epidemiological methods, together with an opportunity to study the medical needs of society. He must be taught the contribution of the behavioural sciences to our understanding of the nature of the society in which medical problems arise and must be solved. The study of epidemiology should include in addition to the spread of diseases such topics as food habits, housing needs, and the medico-social consequences of a longer expectation of life. It is now asserted that modern epidemiology is concerned with all health and illnesses in population groups, including the health services which serve them. Again, the study of the medical needs of society requires a knowledge of the existing services and some appraisal of their efficiency and shortcomings.

Though much of this can be taught within the medical school and as part of the undergraduate's clinical training, for the real significance of the social aspects of health and disease to be appreciated some practical experience in the community must be included. This requires ready access to general practice and the domiciliary services provided by local authorities. Health centres and group practices offering both preventive and curative medicine and the social services run by local health authorities should prove particularly valuable bases for instruction outside hospital. The challenge here is to stimulate the student to be interested in community medicine as well as the care of individual patients. How Newcastle upon Tyne University is meeting this challenge Drs. J. H. Walker and H. G. Barnes report at page 1129 of the B.M.F. this week. ${ }^{2}$ The scheme of undergraduate education they describe includes study of "family medicine and community medicine" on alternate days, and these two aspects of medicine are co-ordinated in carefully prepared seminars.

\footnotetext{
- Report on Departments of Social and Preventive Medicine. Royal College of Physicians. 1966. London.

- See also Brit. med. J., 1966, 2, 292.
}

In the clinical years of the curriculum departments of social medicine should rank in importance with the other major departments. It is also desirable for training in social medicine to be amplified during the preregistration year and afterwards in postgraduate courses when the doctor has had some experience of community care and is anxious for the opportunity to discuss with others the difficulties which arise The social aspects of disease become of increasing importance as community medical care grows. To neglect them in medical training is to run the risk of producing doctors who are not equipped to see the patient as a whole man with medical and related social needs.

The establishment in each medical school of an active and fully recognized department of social and preventive medicine with a full-time head could at this time do much to encourage the integration of medical care and forge the link between the hospital and domiciliary services which is so badly needed.

\section{Necrotizing Enterocolitis in Premature Infants}

An unusual hazard of premature birth is necrotizing enterocolitis. A. Mizrahi and colleagues ${ }^{1}$ recently described 18 cases seen in the Babies Hospital, New York, during a ten-year period. During these years the disease attacked about $1 \%$ of the premature infants, and it caused 16 of the 634 deaths among them.

The condition may be less common in the Anglo-Saxon communities than in Continental Europe. Few British ${ }^{2}$ or American cases have been reported, but $\mathrm{H}$. Willi, ${ }^{3}$ from Switzerland, described 62 cases in 1944, all fatal. Among other Continental reports that of A. Rossier and colleagues described a series of 15 cases in 1959 . The condition is not necessarily fatal if the intestinal perforation, which is common, is recognized and the patient receives surgical treatment. ${ }^{25}$

The aetiology of the enterocolitis is unknown. Though various pathogenic bacterial agents are usually present in the peritoneal fluid or in the blood, they are not consistently found and they may be the result rather than the original cause of the disorder. Antibiotic treatment is usually unsuccessful.

The condition tends to affect the smaller premature infants, most of them having been under $1,500 \mathrm{~g}$. ( $3.3 \mathrm{lb}$.) at birth It has been found in a premature stillborn infant.

Early rupture of the membranes, amnionitis, and fever in the mother were commonly noted. The signs most frequently seen in the infants were a prolonged gastric emptying time, apnoea, jaundice, abdominal distension, vomiting, and gastrointestinal bleeding, in that order of frequency. Later, peritonitis and shock supervene. The disease usually begins during the first 14 days of age, but it has been found up to 3 months. ${ }^{3}$ Case-to-case transmission does not seem to occur, though occasionally cases tend to present in small groups, as any rare disease may do.

The clinical signs are by no means pathognomonic, though the diagnosis may be suspected if the signs point to it in a

\footnotetext{
${ }^{1}$ Mizrahi, A., Barlow, O., Berdon, W., Blanc, W. A., and Sitverman W. A., f. Pediat., 1965, 66, 697.

2 Rickham, P. P., Arch. Dis. Childh., 1955, 30, 23.

- Willi, H., Ann. paediat. (Basel), 1944, $162,87$.

- Rossier, A., Sarrut, S., and Delplanque, J., Ann. Pédiat., 1959, 35 1428

${ }^{5}$ Hyde, G. A., jun., and Santulix, T. V., Pediatrics, 1960, 26, 261.
} 
premature infant weighing less than about $1,500 \mathrm{~g}$. Help may be obtained by radiological investigations. They may show free air in the abdominal cavity, ranging from small amounts to massive pneumoperitoneum, and patterns indicating intestinal obstruction. At this stage blood cultures frequently give a positive result, so that there is strong indication for surgical intervention and for antibiotic treatment by a suitable drug, usually one which is effective against Gramnegative organisms.

The most commonly affected site is the terminal ileum, followed by the ascending and transverse colon. Fibrinoid necrosis of the mucosa is succeeded by necrotizing enteritis, ulceration, and pseudomembraneous inflammation, and is complicated at times by perforations and intestinal pneumatosis. True obstruction of the intestinal tract has not been found.

\section{Diagnosis of Amyloidosis}

Primary amyloid is a rare disease and none of its clinical manifestations is pathognomonic. The most common symptoms are weakness, dyspnoea, ankle oedema, and loss of weight, but unexplained congestive cardiac failure or the nephrotic syndrome appearing in an adult should arouse suspicion of the possibility of underlying amyloid degeneration. In secondary amyloidosis the presence of the predisposing disease, such as tuberculosis or rheumatoid arthritis, usually alerts the clinician. In primary amyloidosis no such help is present. It is not surprising, therefore, that until recently most cases were diagnosed only post mortem.

The Congo red test, introduced by $\mathrm{H}$. Bennhold ${ }^{1}$ over 40 years ago, is of comparatively little help. A considerable quantity of amyloid is necessary to bind a significant amount of the dye, and this produces a high proportion of false negative results. Techniques for obtaining biopsy specimens of the liver, kidney, and other organs provide a far more reliable diagnostic procedure but introduce new difficulties. Aspiration biopsy of abdominal viscera carries a small but nevertheless real risk and should not be used if the necessary information is obtainable by safer methods. For this reason E. Calkins and A. S. Cohen ${ }^{2}$ introduced biopsy of the gingiva, while P. A. Green ${ }^{3}$ and his colleagues used fragments of small intestine. Once again, however, the incidence of false negatives was too high to warrant the general adoption of these methods.

The high incidence of amyloidosis in familial Mediterranean fever has led to a considerable interest in amyloid among clinical investigators in Israel, and in $1960 \mathrm{~J}$. Gafni and E. Sohar ${ }^{4}$ claimed that a positive diagnosis could be obtained in $75 \%$ of cases by biopsy of the rectal mucous membrane. This compares very well with their own figures of $87 \%$ and $48 \%$ for renal and hepatic biopsies respectively. The general adoption of this method throughout Israel almost doubled the rate of antemortem diagnosis of amyloid degeneration, so that in 1959 a rate of $74 \%$ was achieved, compared with $39 \%$ for the previous eight years.

R. A. Kyle, R. J. Spencer, and D. C. Dahlin ${ }^{5}$ have recently drawn further attention to the value of rectal biopsy in the

\footnotetext{
1 Bennhold, H., Disch. Arch. klin. Med., 1923, 142, 32.

Calkins, E., and Cohen, A. S., Bull. rheum. Dis., 1960, 10, 215.

- Green, P. A., Higgins, J. A., Brown, A. L., iun., Hoffman, H. N., and Sommerville, R. L., Gastroenterology, 1961, 41, 452.

4 Gafn, J., and Sohar, E., Amer. F. med. Sci., 1960, 240, 332.

- Kyle, R. A., Spencer, R. J., and Dahlin, D. C., ibid, 1966, 251, 501
}

diagnosis of primary amyloid. In 20 cases at the Mayo Clinic a definite diagnosis was made by rectal biopsy in 17, and of the 3 false negative results 2 were attributable to an inadequate biopsy specimen. Apparently the submucosa must be included in the specimen, for on 13 occasions the deposits of amyloid were found only in that layer or in the muscularis mucosae and not in the mucous membrane itself. In 79 cases suspected of primary amyloidosis on clinical grounds rectal biopsy was negative. Not one of these has so far shown evidence of the disease, though some have died and necropsy has been carried out, others have had biopsy examinations of other organs, and many have been followed up for several years. This convincing confirmation of the value of rectal biopsy must now make it the method of choice in all patients suspected of having primary amyloidosis.

\section{Medicine in the Tropics}

The practice of medicine in tropical countries differs from that in Britain in many ways. A doctor going out to Africa, for instance, needs to do more than learn the features of malaria and sleeping-sickness. The differential diagnosis of common conditions such as heart failure and abdominal pain requires a reorientation of thought: "common diseases commonly occur," but there is wide variation in the diseases that are common in different countries and among different races. At page 1119 of this week's B.M.F. appears the first of a series of articles on medicine in the tropics. Others will be published at intervals of about a month. These articles will describe diseases and syndromes as seen in the tropics rather than tropical diseases as seen in Britain. The series should help doctors working in tropical countries and particularly those who are working single-handed or on isolated stations. The articles will also be of interest to doctors in Britain who are contemplating work in the developing countries. Besides this, B. Maegraith ${ }^{1}$ has recently drawn attention to the "shrinking world," the result of modern travel, in which there is no exotic disease that may not present in practice in Britain either in a traveller or in an immigrant. The world can now be encircled easily within the incubation period of most of the quarantinable diseases. $^{2}$

In the articles the emphasis will be on practical guidance on diagnosis and management. All clinical investigations and laboratory procedures mentioned will be described in enough detail for a doctor to carry them out without skilled assistance. Treatment will also be described in detail, the dosage and duration of administration of drugs will be stated, and the problems that arise in the management of patients in underdeveloped rural communities will be discussed.

For centuries British doctors have studied and practised tropical medicine. Maegraith's shrinking world is making practical knowledge of tropical disease more and more essential for all doctors, wherever they work. The articles in this series are written by experts with first-hand knowledge of their subjects, but each one will be describing disease as it occurs in only one portion of the world. We hope that this series will stimulate interest in this challenging field, and perhaps provoke correspondence from others with different experience. 